\title{
Virtual Sensing of Wheel Position in Ground-Steering Systems for Aircraft Using Digital Twins
}

\author{
Mattia Dal Borgo, Stephen J. Elliott, Maryam Ghandchi Tehrani, and Ian M. Stothers ${ }^{1}$ \\ Institute of Sound and Vibration Research \\ University of Southampton, SO17 1BJ, Southampton, United Kingdom \\ ${ }^{1}$ Ultra Electronics Precision Control Systems \\ St Johns Innovation Park, CB4 0DS, Cambridge, United Kingdom
}

\begin{abstract}
The ground-steering system is a part of the nose landing gear, which is fundamental to an aircraft's safety. A sensing mechanism estimates the wheel direction, which is then fed back to the controller in order to calculate the error between the desired steering angle and the actual steering angle. As in many safety-critical control systems, the sensing mechanism for the nose wheel direction requires the use of multiple redundant sensors to estimate the same controlled signal. A virtual sensing technique is commonly employed, which estimates the steering angle using the measurements of multiple remote displacement sensors. The wheel position is then calculated on the basis of the nonlinear alignment of these sensors.

In practice, however, each sensor is subject to uncertainty, minor and major faults and there is also ambiguity associated with the estimate of the steering angle because of the geometric nonlinearity. The redundant sensor outputs are thus different from each other, and it is important to reliably estimate the controlled signal under these conditions.

This paper presents the development of a digital twin of the ground-steering system, in which the effect of uncertainties and faults can be systematically analysed. A number of state estimation algorithms are investigated under several scenarios of uncertainty and sensor faults. Two of these algorithms are based on a least squares estimation approach, the other algorithm, instead, calculates the steering angle estimate using a soft-computing approach. It is shown that the soft-computing estimation algorithm is more robust than the least squares based methods in the presence of uncertainties and sensor faults. The propagation of an uncertainty interval from the sensor outputs to the steering angle estimate is also investigated, in order to calculate the error bounds on the estimated controlled signal. The optimal arrangement of the sensors is also investigated using a parametric study of the uncertainty propagation, in which the optimal model parameters are the ones that generates the smallest uncertainty interval for the estimate.
\end{abstract}

Keywords: Digital Twin, Virtual Sensing, Aircraft Ground-Steering System, Soft-Computing, Redundant Sensors

\section{INTRODUCTION}

The ground-steering system is a part of the nose landing gear, which is fundamental to an aircraft's safety. A ground-steering system commonly consists of two linear actuators (electric or hydraulic), a power supply, a control valve, a follow-up device between the gear and the steering collar, a sensing mechanism, a control valve, a pilot control (switch) and a pilot-operated steering wheel, which can be either a bar or the rudder pedals or a combination of them [1]. The feedback signal of the ground-steering control loop is the steering angle of the nose wheel, which is then used to calculate the error between the desired steering angle and the actual steering angle.

Although the wheel direction cannot usually be measured directly with an angular sensor, a virtual sensing technique is adopted, which estimates the steering angle using redundant measurements remote from the position of interest. Multiple linear displacement sensors are arranged on a mechanism that is similar to the dual push-pull steering mechanism [2] and the 
nonlinear geometry of the sensor arrangement is used to calculate the wheel angular position. If the sensors were to operate perfectly, only a subset of them would need to be used for the estimation. In practice, however, the sensors are subject to uncertainty, minor or major faults and their operation may be nonlinear. Therefore, it is crucial to reliably estimate the controlled signal under these conditions, assessing the degree of confidence with which each sensor should be treated.

This paper presents the development of a digital twin of the ground-steering system, in which the effect of uncertainties and faults can be systematically analysed. Three state estimation algorithms are discussed that calculate the steering angle estimate under several scenarios including model uncertainty, measurement noise and sensor faults.

The paper is organized as follows. The development of the digital twin is first presented in section 2. An interval type of uncertainty in the sensor outputs is then introduced in section 3 in order to calculate the propagated uncertainty interval in the steering angle estimation. Three state estimation algorithms are investigated in section 4 , two of which are least squares based approaches and the remaining one is a soft-computing method. The results of the comparison among the estimation algorithms in terms of estimation accuracy when the digital twin is affected by model uncertainty, sensor noise and potential sensor faults, are presented in section 5 . The conclusions are summarized in section 6 .

\section{DIGITAL TWIN OF THE GROUND-STEERING SYSTEM}

The steering angle is estimated using a virtual sensing technique, which consists of multiple linear variable differential transformer (LVDT) sensors remotely located from the position of interest. The wheel direction is then calculated based on the nonlinear geometry of the sensor alignment. A model of the ground-steering sensing mechanism is shown in Figure 1, in which four LVDTs are used, two on the left arm and two on the right arm. The model parameters, which are given in Table 1, have been selected according to the study in [2].

According to the schematic in Figure 1, the controlled signal $\alpha$ is estimated from the measurements of the left $\left(d_{L 1}, d_{L 2}\right)$ and right $\left(d_{R 1}, d_{R 2}\right)$ LVDTs, respectively. In practice, however, the model parameters of the sensing mechanism are subject to uncertainty, as well as the sensor outputs. The LVDTs can also be affected by minor or major faults and there is ambiguity associated with all of the sensors due to the nonlinearity. The digital twin of the ground-steering system consists of the sensing mechanism model together with the uncertainties associated with its parameters, the noise in the sensor outputs and the potential faults in the LVDTs.

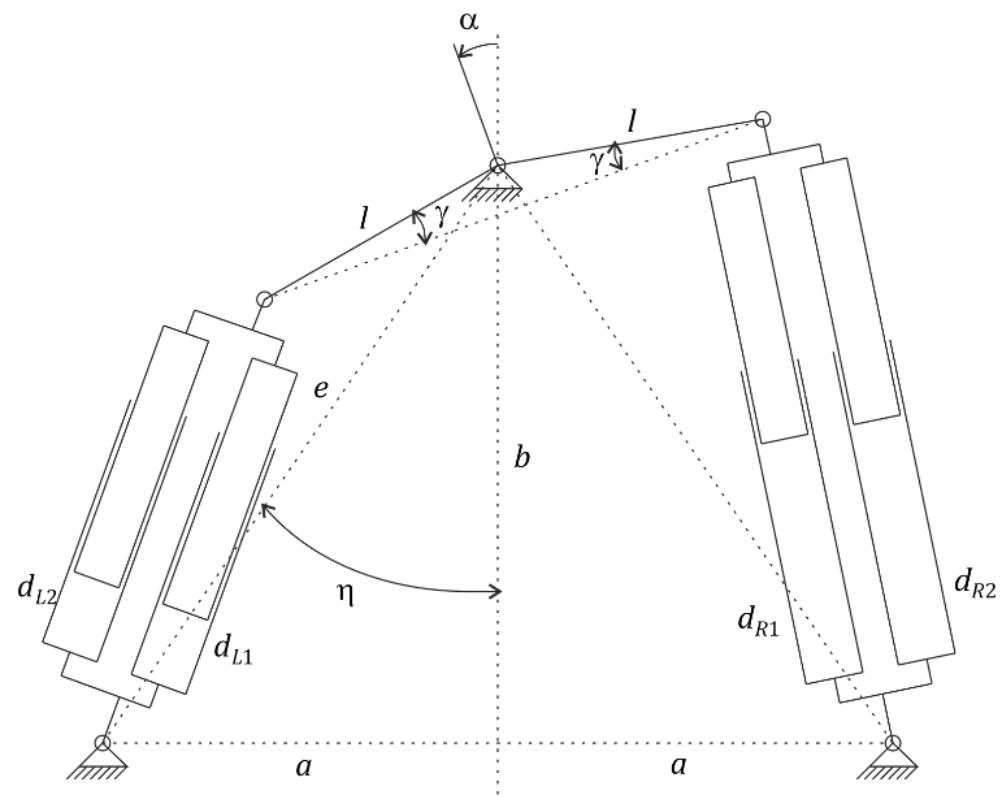

Figure 1: Schematic of the ground-steering sensing mechanism for the steering angle estimation, which includes the 4 LVDT sensors. 
The nonlinear relationship between the wheel direction and the outputs from the LVDTs can be studied through a kinematic analysis of position in which it is assumed that the model parameters are exact and the output from the sensors are ideal, hence without any noise of faults.

Table 1: Assumed model parameters, according to the schematic of Figure 1.

\begin{tabular}{|c|c|c|}
\hline Parameter & Value & Units \\
\hline$e$ & 200 & $\mathrm{~mm}$ \\
\hline$l$ & 153 & $\mathrm{~mm}$ \\
\hline$\eta$ & 44 & $\mathrm{deg}$ \\
\hline$\gamma$ & 26 & $\mathrm{deg}$ \\
\hline
\end{tabular}

The derivation of the relationship between the steering angle and the sensor outputs can be found in [3] and is omitted in this paper. However, it results in the following set of equations,

$$
\left\{\begin{array}{l}
d_{L i}^{2}=l^{2}+a^{2}+b^{2}-2 l[a \cos (\alpha+\gamma)+b \sin (\alpha+\gamma)] \\
d_{R i}^{2}=l^{2}+a^{2}+b^{2}-2 l[a \cos (\alpha-\gamma)-b \sin (\alpha-\gamma)]
\end{array},\right.
$$

in which $\alpha$ is the steering angle, $\left(d_{L i}, d_{R i}\right)$ are the sensor outputs and $l, a, b, \gamma$ are the model parameters. The relationship between the outputs from the LVDTs and the steering angle defined by Eq. (1) is also shown by the solid red line in Figure 2(a). Since it was assumed that the sensor were ideal, hence without being affected by noise and faults, both right LVDTs give the same output and both left LVDTs give the same output. In this case only a subset of the four sensors would be needed to calculate the steering angle. A couple of one left and one right sensor measurements will determine a unique value of the steering angle, as shown in Figure 2(a). The red line in Figure 2(a) can be projected onto the x-y plane, which results on the black solid line shown in Figure 2(b). This curve relates a subset of left and right sensor measurements to its unique steering angle value. However, if only a left or a right sensor output was used, there would be ambiguity on the value of the actual steering angle, because the nonlinear relationship between the steering angle and each sensor output generates double solutions. When both left and right steering angle are used, only two out of the four possible solutions are recurring and can be considered as the actual steering angle.

The set of Eqs. (1) can be rewritten in an explicit form in order to solve the inverse problem of determining the unknown steering angle from the known sensor outputs. Hence, Eq. (1) becomes,

$$
\left\{\begin{array}{l}
\sin (\alpha)[b \cos (\gamma)-a \sin (\gamma)]+\cos (\alpha)[a \cos (\gamma)+b \sin (\gamma)]-\frac{l^{2}+a^{2}+b^{2}-d_{L i}^{2}}{2 l}=0 \\
\sin (\alpha)[a \sin (\gamma)-b \cos (\gamma)]+\cos (\alpha)[a \cos (\gamma)+b \sin (\gamma)]-\frac{l^{2}+a^{2}+b^{2}-d_{R i}^{2}}{2 l}=0
\end{array} .\right.
$$

Given a pair of measurements $\left(d_{L}, d_{R}\right)$, the steering angle estimate can be found by solving Eq. (2) by iteration using for example Newton-Raphson method. In this ideal scenario without uncertainties and faults, the solution of Eq. (2) generates the same solution of Eq. (1), which is shown with the red line in Figure 2(a). 


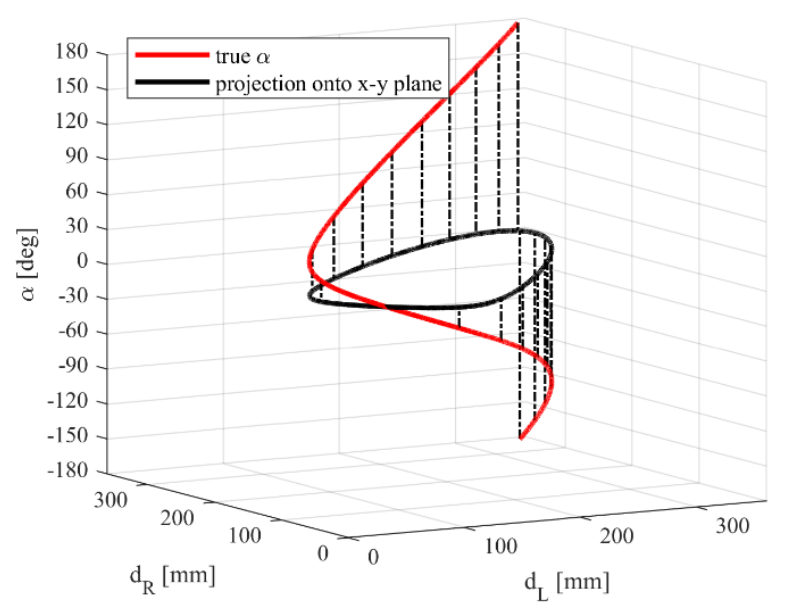

(a)

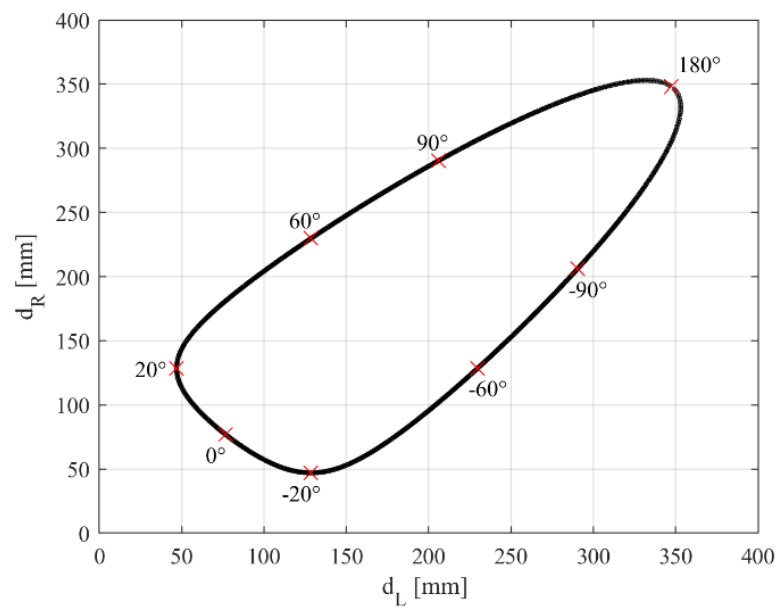

(b)

Figure 2: (a) Variation of the steering angle with respect to the left and right sensor outputs. The true steering angle is shown with the red solid line $\left(\_\right.$) and its projection to the $\mathrm{x}-\mathrm{y}$ plane with the black solid line ( displacements $\left(d_{R}, d_{L}\right)$; each set of right and left sensor output correspond to a unique value of the steering angle.

\section{INTERVAL UNCERTAINTY PROPAGATION}

In practice, the outputs from the sensors deviate from the ideal values because they are subject to measurement noise and faults. In this section we assume that none of the sensors is faulty, but all of them are subject to an interval type of uncertainty around the true measurement values. The output intervals from the sensors can then be written as,

$$
\left[d_{L i}\right]=\left[\begin{array}{ll}
\underline{d_{L i}} & \overline{d_{L i}}
\end{array}\right],\left[d_{R i}\right]=\left[\begin{array}{ll}
\underline{d_{R i}} & \overline{d_{R i}}
\end{array}\right],
$$

where $\underline{d}=\mu_{d}-\Delta_{d}$ is the lower bound and $\bar{d}=\mu_{d}+\Delta_{d}$ is the upper bound of the uncertainty interval, whereas [d] denotes the interval of possible values with an unspecified probability density function [4]. The interval [d] has a mean value $\mu_{d}$ and an uncertainty amplitude $\Delta_{d}$. According to Eqs. $(1,2)$, an uncertainty on the sensor outputs causes an uncertainty on the steering angle, which can be written as,

$$
[\alpha]=[\underline{\alpha} \quad \bar{\alpha}],
$$

where $\underline{\alpha}=\mu_{\alpha}-\Delta_{\alpha, l}$ and $\bar{\alpha}=\mu_{\alpha}+\Delta_{\alpha, u}$ are the lower and upper bounds of the steering angle interval $[\alpha]$. The mean value of the steering angle interval is denoted as $\mu_{\alpha}$, whereas, $\Delta_{\alpha, l}$ and $\Delta_{\alpha, u}$ are the lower and upper bounds of the propagated uncertainty, respectively.

Figure 1 shows, with the thick black lines, the left and right LVDT outputs with respect to the steering angle, according to Eq. (1). The thinner horizontal black lines represent interval uncertainties around an admissible measurement pair $\left(d_{L}, d_{R}\right)$, which is shown with green lines. The uncertainty bounds shown in Figure 1 have been set to $\Delta_{d}= \pm 10 \mathrm{~mm}$ for each sensor for visual clarity. 


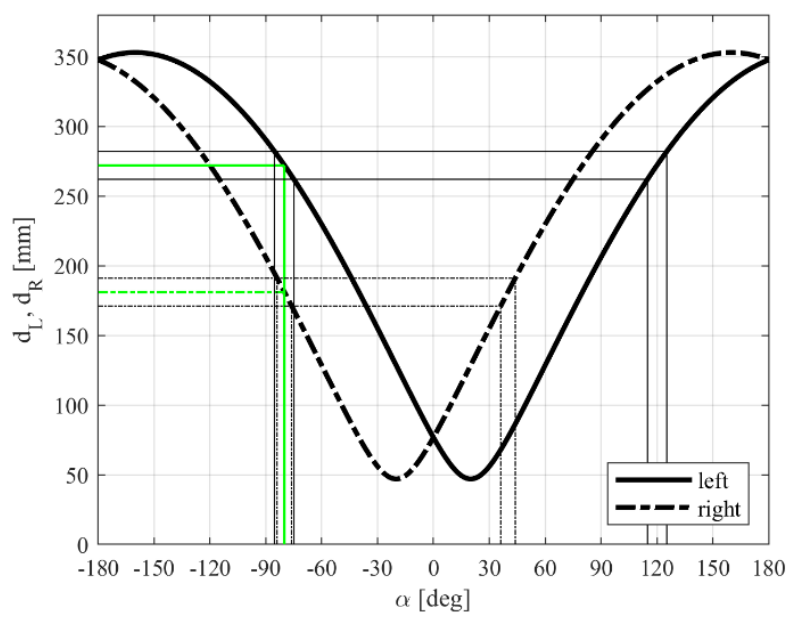

Figure 3: Position of the left ( _ $)$ and right ( _ - - ) sensors versus the steering angle. Propagation of an uncertainty interval around a true measurement value ( $\left.\_,--\right)$. The interval uncertainty has been set to a large value $\left(\Delta_{d}= \pm 10 \mathrm{~mm}\right)$ for visual clarity.

Each uncertainty interval associated with each sensor output generates two uncertainty intervals on the steering angle. All the propagated uncertainty intervals can be combined to calculate the smallest uncertainty interval around the true value of the steering angle. The upper and lower bounds of the propagated uncertainty interval around the true steering angle are shown in Figure 4 for all steering angles between $-90^{\circ}$ and $+90^{\circ}$. The upper and lower error bounds on $\Delta_{\alpha}$ scale approximately linearly on the magnitude of $\Delta_{d}$, up to $\Delta_{d}= \pm 20 \mathrm{~mm}$.

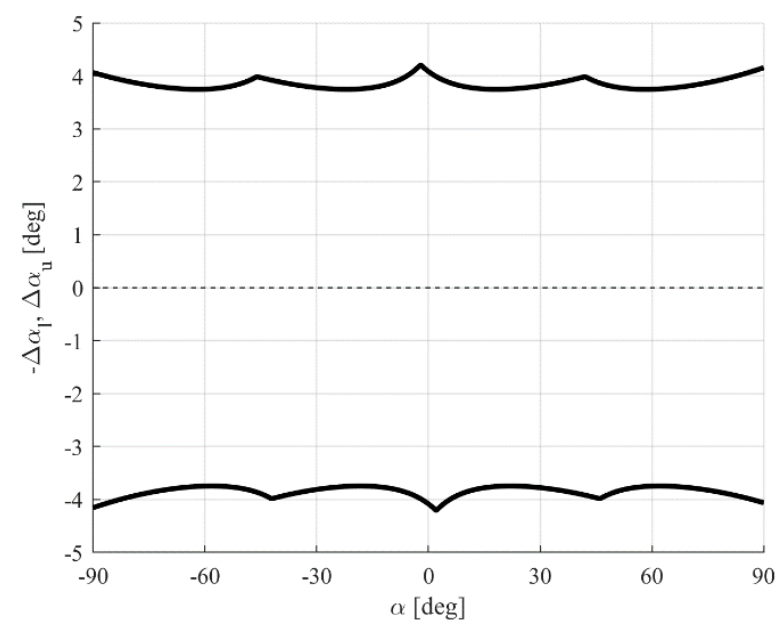

Figure 4: Lower an upper bounds of the error in the estimation of the steering angle, $\Delta_{\alpha, l}$ and $\Delta_{\alpha, u}$, for an interval uncertainty in the sensors of $\Delta_{d}= \pm 10 \mathrm{~mm}$. The propagated uncertainty scales approximately linearly with $\Delta_{d}$ up to $\Delta_{d}= \pm 20 \mathrm{~mm}$.

The geometric parameters presented in Table 1 can be varied in a parametric study to investigate how they affect the propagated uncertainty. Figure 5(a,b,c,d) show the influence of the parameters $e, \eta, l, \gamma$ on the propagated uncertainty, respectively. The contour plots of Figure 5 show only the upper bound of the uncertainty interval, as this is symmetric with respect to the true value of the steering angle. For each design parameter, an optimal value can be determined, which generates the smallest interval of propagated uncertainty. The optimal values of the parameters are indicated with the red lines in Figure 5(a,b,c,d). The ground-steering sensing mechanism can then be redesigned using the newly determined values of the sensor arrangements. This guarantees a smaller propagation of uncertainties from the sensors to the estimated steering angle. It should be noticed that the parameters $e, \eta$ and $\gamma$ indicated in Table 1 are very close to the optimal parameters shown 
in Figure 5(a,b,d). The optimal parameter $l$, instead, is larger than the one appearing in the table, but the values of the propagated uncertainty are very close to each other, making this difference almost negligible.

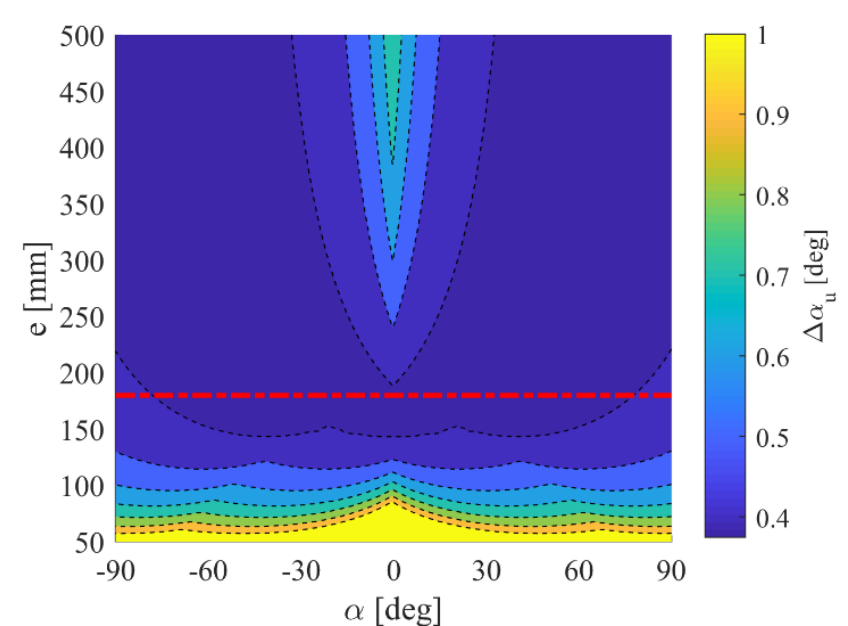

(a)

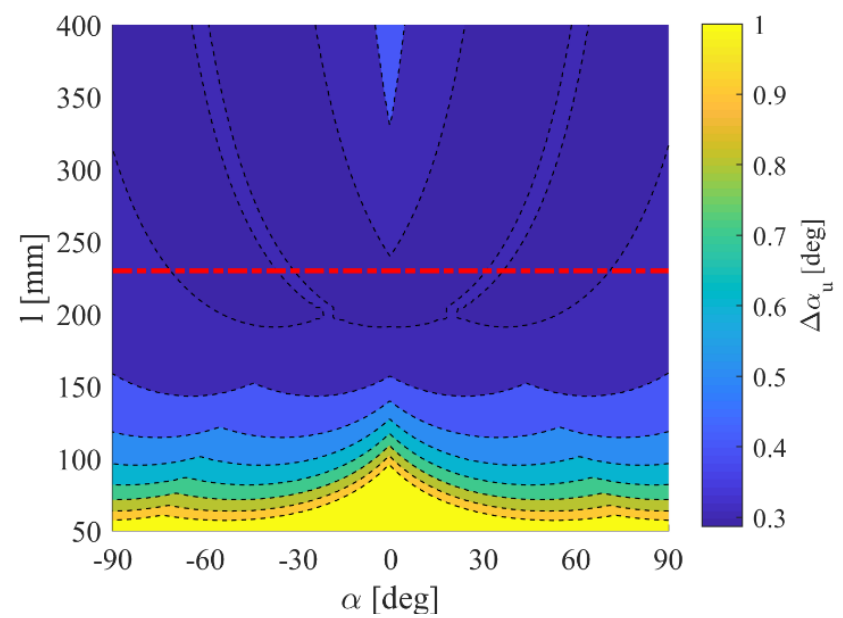

(c)

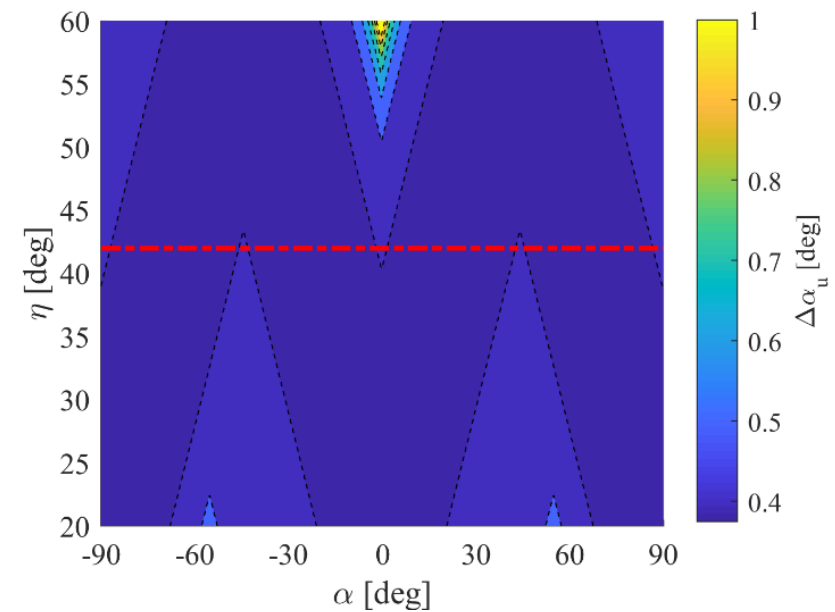

(b)

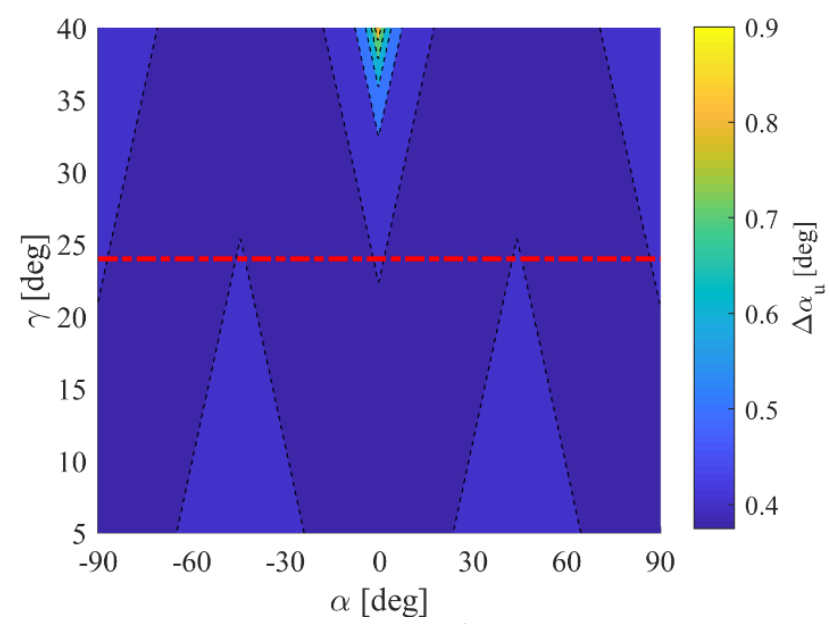

(d)

Figure 5: Parametric analysis of the propagated uncertainty interval with respect to the values of the model parameters of Table 1.

Maximum error in the steering angle estimation for a measurement uncertainty $\Delta_{d}= \pm 1 \mathrm{~mm}$ : (a) varying $e$; (b) varying $\eta$; (c) varying

$l$; (d) varying $\gamma$. The red dashed lines represent the optimal values of the parameters that generate the smallest uncertainty interval on the steering angle.

\section{STATE ESTIMATION ALGORITHMS}

The previous section dealt with the measurement uncertainty propagation, however, the true value of the steering angle was assumed to be known. In practice, the steering angle is the unknown variable and the only variables that are known are the outputs from the LVDTs and the model parameters. Both the model parameters and the sensor outputs, however, are affected by uncertainties on their values, and the sensors are subject to potential faults. Hence, it is crucial to reliably estimate the controlled signal $\alpha$ and to calculate the level of uncertainty associated with this estimation. This section presents a comparison among three different algorithms for the estimation of the steering angle. The first algorithm is based on an ordinary least-squares (OLS) approach that is commonly implemented in Global Positioning Systems (GPS), where the position of a GPS receiver is calculated using the signals sent by a set of redundant satellites [5, 6]. For the OLS estimation algorithm, the set of Eqs. (2) can be rewritten in matrix form as follows,

$$
\mathbf{A} \hat{\mathbf{x}}=\mathbf{d}-\hat{\mathbf{v}}
$$


which is also known as observation equation, where

$$
\mathbf{A}=\left[\begin{array}{ll}
(b \cos \gamma-a \sin \gamma) & (a \cos \gamma+b \sin \gamma) \\
(b \cos \gamma-a \sin \gamma) & (a \cos \gamma+b \sin \gamma) \\
(a \sin \gamma-b \cos \gamma) & (a \cos \gamma+b \sin \gamma) \\
(a \sin \gamma-b \cos \gamma) & (a \cos \gamma+b \sin \gamma)
\end{array}\right]
$$

is the matrix of model parameters,

$$
\hat{\mathbf{x}}=\left\{\begin{array}{l}
\sin \hat{\alpha} \\
\cos \hat{\alpha}
\end{array}\right\}
$$

is the vector of estimated states,

$$
\mathbf{d}=\frac{1}{2 l}\left\{\begin{array}{l}
l^{2}+a^{2}+b^{2}-d_{L 1}^{2} \\
l^{2}+a^{2}+b^{2}-d_{L 2}^{2} \\
l^{2}+a^{2}+b^{2}-d_{R 1}^{2} \\
l^{2}+a^{2}+b^{2}-d_{R 2}^{2}
\end{array}\right\},
$$

is the vector containing the measurements, also known as observation vector, and $\hat{\mathbf{v}}$ is the error vector between the estimated and the measured observations. The OLS estimate is computed by minimizing the sum of the squared errors, which results in,

$$
\widehat{\mathbf{x}}=\left(\mathbf{A}^{\mathbf{T}} \mathbf{A}\right)^{-\mathbf{1}} \mathbf{A}^{\mathbf{T}} \mathbf{d},
$$

And the steering angle estimation is derived from Eq. (9) as follows,

$$
\hat{\alpha}=\operatorname{atan}\left(\frac{\sin \hat{\alpha}}{\cos \hat{\alpha}}\right)
$$

The OLS estimator is computationally inexpensive and provides accurate enough estimates with noisy measurements, however, it lacks of robustness in presence of larger deviations or faults in the sensors. This motivates to look for estimation algorithms that are more robust against outliers. Given the four measurements from the left and right LVDTs, eight possible steering angles are calculated by solving the inverse kinematic problem given in Eq. (2). The solutions given by the left sensors can be written as,

$$
\hat{\alpha}_{L i, 1,2}=2 \operatorname{atan}\left(\frac{b \pm \sqrt{a^{2}+b^{2}-L_{i}^{2}}}{a+L_{i}}\right)-\gamma
$$

and for the right sensors as,

$$
\hat{\alpha}_{R i, 1,2}=2 \operatorname{atan}\left(\frac{-b \pm \sqrt{a^{2}+b^{2}-R_{i}^{2}}}{a+R_{i}}\right)+\gamma
$$

where $L_{i}$ and $R_{i}$ have been defined as,

$$
L_{i} \triangleq \frac{l^{2}+a^{2}+b^{2}-d_{L i}^{2}}{2 l}, R_{i} \triangleq \frac{l^{2}+a^{2}+b^{2}-d_{R i}^{2}}{2 l}
$$

The eight possible steering angle estimates cast the observation vector as follows, 


$$
\widehat{\boldsymbol{\alpha}}=\left\{\begin{array}{llllllll}
\hat{\alpha}_{L 1,1} & \hat{\alpha}_{L 1,2} & \hat{\alpha}_{L 2,1} & \hat{\alpha}_{L 2,2} & \hat{\alpha}_{R 1,1} & \hat{\alpha}_{R 1,2} & \hat{\alpha}_{R 2,1} & \hat{\alpha}_{R 2,2}
\end{array}\right\} .
$$

One of the most common methods of robust estimation are M-estimators, which can be regarded as a generalization of the OLS with a higher tolerance for outliers. In this case, instead of using the sum of the squared errors, the cost function can be written as [7],

$$
\sum_{i=1}^{n} \rho\left(e_{i}\right),
$$

where $\rho(e)$ is a general function of the error. Solving Eq. (15) is equivalent to solve a weighted least squares problem. However, in this case, the weights depend upon the errors, the errors depend upon the estimates, and the estimates depend upon the weights. Hence, an iterative approach is required, which is called iteratively reweighted least squares (IRLS) [7]. The IRLS algorithm works as follow,

- Select an initial estimate, such as the OLS estimate, or the median estimate;

- At each iteration calculate the error residuals $e_{i}$ and associate weights from the previous iteration;

- Solve for the new weighted least squares estimate,

$$
\hat{\alpha}=\left(\mathbf{H}^{\mathbf{T}} \mathbf{W} \mathbf{H}\right)^{-1} \mathbf{H}^{\mathbf{T}} \mathbf{W} \widehat{\boldsymbol{\alpha}},
$$

where $\widehat{\boldsymbol{\alpha}}$ is given by Eq. (14), $\mathbf{H} \in \mathbb{R}^{8 \times 1}$ with all elements of $\mathbf{H}$ equal to $1 / 8$, and $\mathbf{W}$ is a diagonal matrix containing the weights;

- The previous two steps are repeated until the estimate converges.

Different $\rho(e)$ can be used for the IRLS algorithm, the one used in this paper is the Huber objective function [7], for which the weight become,

$$
w\left(e_{i}\right)= \begin{cases}1 & \left|e_{i}\right| \leq k \\ k /\left|e_{i}\right| & \left|e_{i}\right|>k\end{cases}
$$

where $k$ is a tuning constant, which has been chosen to be twice the median absolute deviation (MAD) in this study. The estimator is more robust against outliers for smaller values of $k$.

Although the IRLS is more robust against outliers with respect to the OLS, it is not always able to deal with sensor faults, because in certain circumstances more than half of the elements of Eq. (14) are incorrect and far from the true value of the steering angle. A different approach for the estimation of the steering angle is the soft-computing (S-C), or soft-voting method. The S-C approach is a sensor management technique that is similar to the majority voting approach, but in this case the observations are assigned weights that are based on fuzzy membership functions and the final estimate is computed as a weighted average of all valid observations. The S-C algorithm can be summarized as follows [8, 9]. Each element of the observation vector given by Eq. (14) is assigned a weight and the consolidated steering angle estimate is the weighted average of all valid observations,

$$
\hat{\alpha}=\sum_{i=1}^{n} w_{i} \hat{\alpha}_{i},
$$

where $w_{i}$ denotes the weight assigned to the observation $\alpha_{i}$ and $n$ is the number of valid observations. The weights $w_{i}$ are calculated as,

$$
w_{i}=\frac{\mu_{i}}{\sum_{j=1}^{n} \mu_{j}},
$$

where $\mu_{i}$ is the membership degree of $\alpha_{i}$ and is bounded between zero and one. The membership degree of each signal is computed as shown in Figure 6, where the current value of each observation (red vertical line) forms the centre of its 
corresponding membership function, which is shown with the black solid line. The membership degree of the signal is the largest membership degree of the remaining valid observations (green vertical lines) according to,

$$
\mu_{i}=\max _{i \neq j} \mu_{i}\left(\hat{\alpha}_{j}\right)
$$

There are various shapes for the membership functions, such as triangular, trapezoidal, sigmoidal or polynomial. A polynomial membership function has been used in this paper, which has a flat top between $\pm 2^{\circ}$ and is null for observation differences above $7^{\circ}$. This membership function is shown in Figure 6.

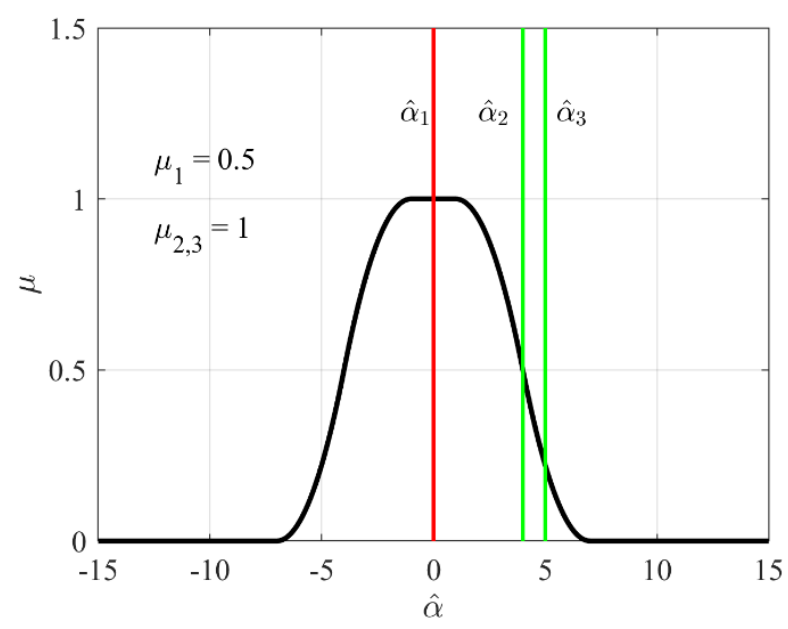

Figure 6: Soft-computing (S-C) membership function. The current value of each observation (red line) forms the centre of its corresponding membership function (black line), which is then used to determine the membership degree of this observation using the other valid observations (green lines).

The implementation of the S-C algorithm is illustrated in Figure 7. The vector of observations is split into the eight observation signals that are then sorted. The membership degrees of the signals are computed according to Eq. (20), reorganized in the original order and combined in a vector. Finally, the consolidated steering angle estimation is computed according to Eqs. $(18,19)$.

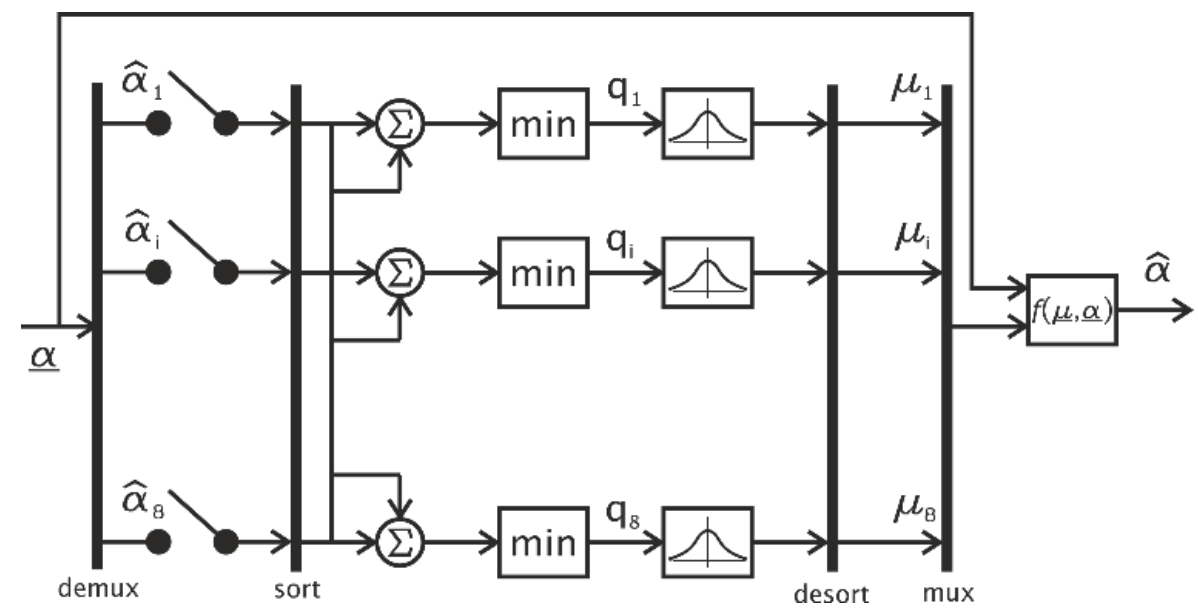

Figure 7: Schematic of the soft-computing algorithm. 


\section{RESULTS}

The methods of OLS, IRLS and S-C are compared in this section for the following scenarios,

- First, the ideal scenario in which both the model parameters and the sensor outputs are assumed to be known perfectly. The results of implementing the OLS, IRLS and S-C approaches are shown in Figure 8(a,b) with the dash-dotted red, dashed green and solid blue line, respectively. The black solid line represents the true value of the steering angle.

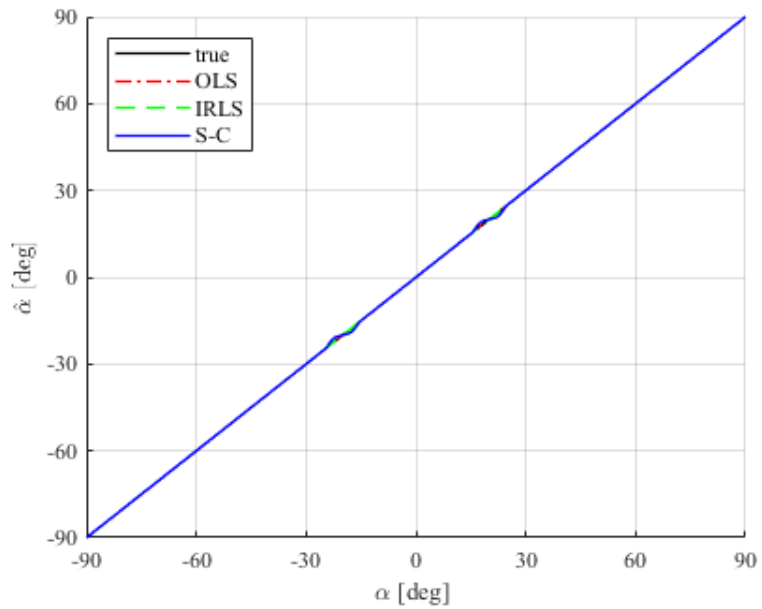

(a)

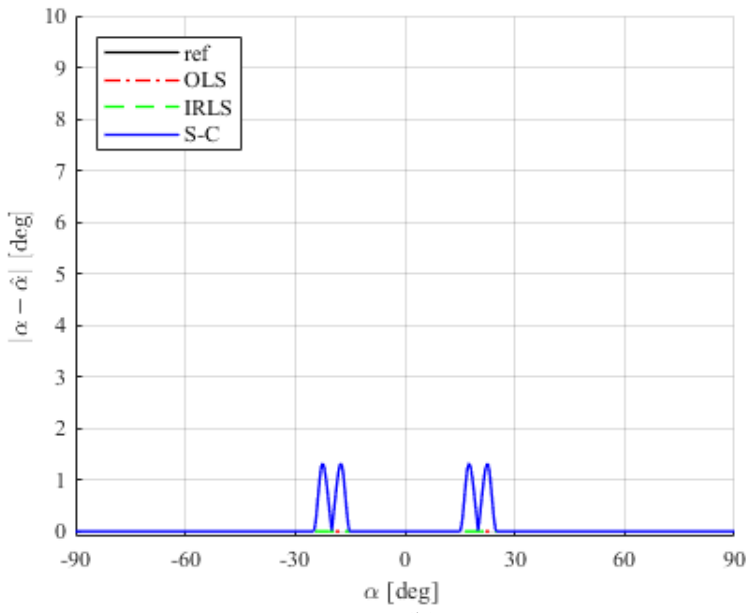

(b)

Figure 8: Comparison amongst estimation algorithms for an ideal scenario. (a) Estimated steering angle; (b) Accuracy of the estimation.

- The second scenario includes an uncertainty of $\Delta= \pm 2 \mathrm{~mm}$ for the model parameters given in Table 1 , and measurement noise of $\Delta_{d}= \pm 2 \mathrm{~mm}$ for the LVDT sensors, however, faults have not been taken into account in this scenario. The results are shown in terms of estimated steering angle and estimation accuracy in Figure 9(a,b).

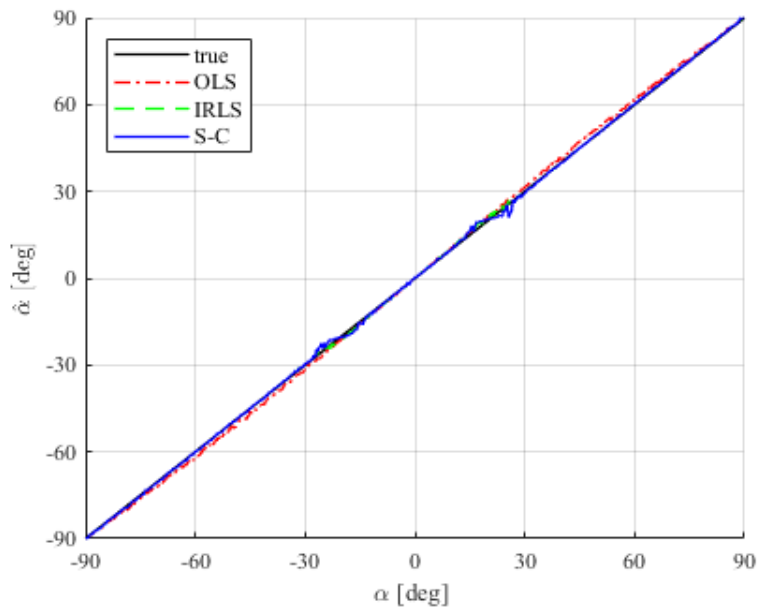

(a)

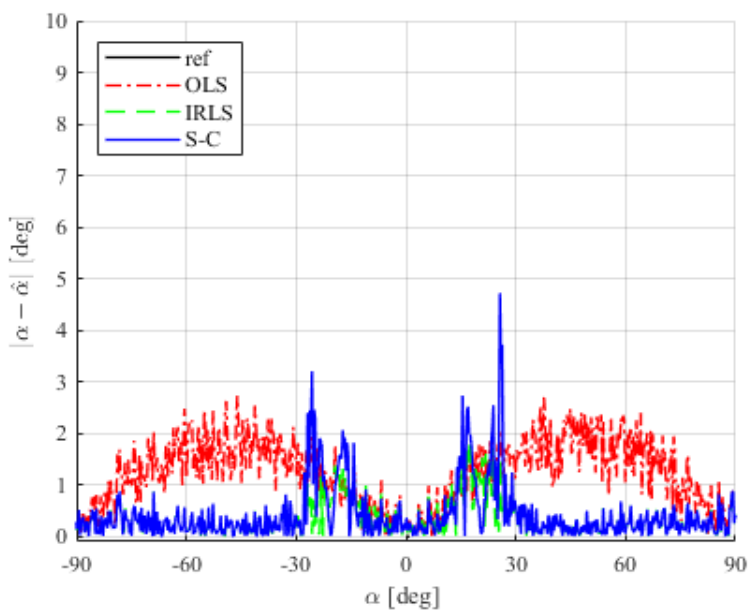

(b)

Figure 9: Comparison amongst estimation algorithms including uncertainty in the model parameters and measurement noise of $\Delta= \pm 2 \mathrm{~mm}$ and $\Delta_{d}= \pm 2 \mathrm{~mm}$, respectively. (a) Estimated steering angle; (b) Accuracy of the estimation.

- The third and final scenario includes parameter uncertainties $(\Delta= \pm 2 \mathrm{~mm})$, measurement noise $\left(\Delta_{d}= \pm 2 \mathrm{~mm}\right)$, and sensor faults. In this case, the faulty sensor id one of the left LVDTs, $d_{L 1}$, which is locked at $120 \mathrm{~mm}$. The results and comparison amongst the different estimation algorithms are shown in terms of estimated steering angle and estimation accuracy in Figure 10(a,b). 


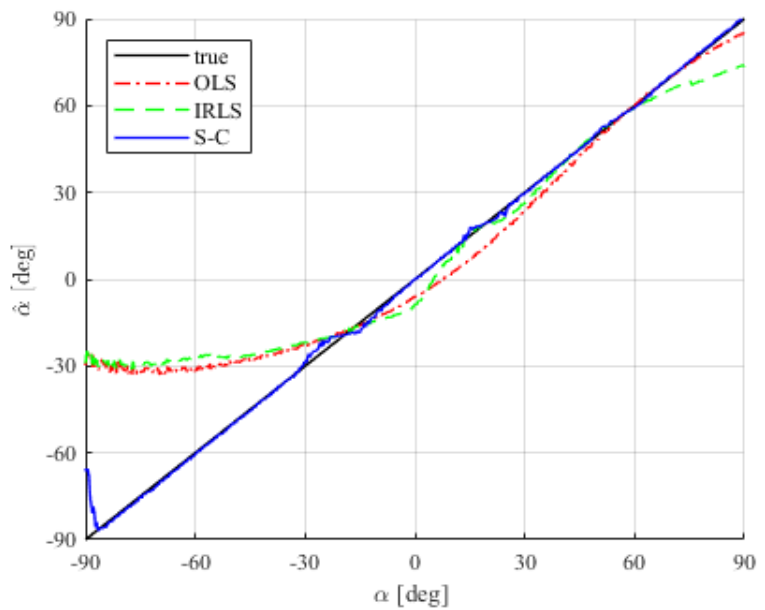

(a)

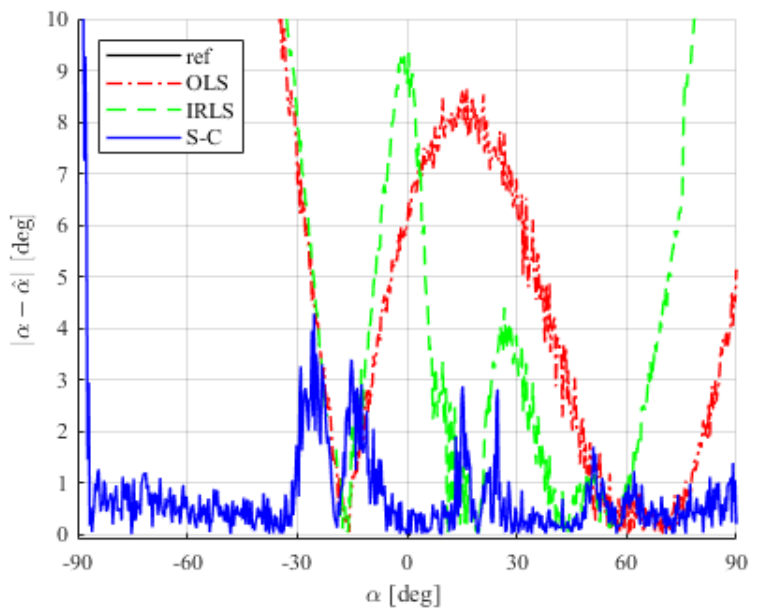

(b)

Figure 10: Comparison amongst estimation algorithms including uncertainty in the model parameters, measurement noise of $\Delta= \pm 2 \mathrm{~mm}$ and $\Delta_{d}= \pm 2 \mathrm{~mm}$, respectively and a faulty sensor $d_{L 1}=120 \mathrm{~mm}$. (a) Estimated steering angle; (b) Accuracy of the estimation.

The results presented in Figure 8 and Figure 9 show that the least squares based estimators can have a slightly better accuracy than the S-C algorithm when none of the sensors is faulty, regardless of the uncertainty. Figure 10, however, show that the SC approach is more robust to sensor faults when compared to the OLS and the IRLS. In fact, it is the only one of the three proposed methods to give a consistent estimation accuracy across all the scenarios that have been studied.

\section{CONCLUSION}

This paper presented the estimation of wheel direction in ground-steering systems for aircraft using the measurements of remote and redundant LVDT sensors subject to uncertainties and faults. Firstly, the digital twin of the ground steering system has been introduced, which consists of a kinematic model of the sensing mechanism together with the model uncertainties, the measurement noise and the potential sensor faults. The nonlinear relationship between the steering angle and the sensor outputs was then discussed.

Secondly, an interval type of uncertainty was introduced for each LVDT output and was propagated through the model to calculate the maximum error in the steering angle estimation. The variation of such error with the wheel position was also presented. A parametric study of the uncertainty propagation was carried out in order to calculate the optimal arrangement of the sensors that generates the smallest error in the estimation of the steering angle.

Finally, three estimation algorithms were presented for the estimation of the wheel direction in presence of model uncertainties, measurement noise and sensors faults. Two least squared based approaches, namely ordinary least squares (OLS) and iteratively reweighted least squares (IRLS), and a soft-computing (S-C) method were introduced. A comparison among these three methods under different scenarios of uncertainties and faults was carried out in terms of accuracy of the estimation for a steering angle ranging from $-90^{\circ}$ to $+90^{\circ}$. It is shown that the S-C estimation algorithm is more robust than the least squares based methods in presence of uncertainties and sensor faults. Future work will relate to the definition of a metric that calculates the degree of confidence in the estimate and the monitoring of the sensor conditions.

\section{ACKNOWLEDGEMENTS}

The authors gratefully acknowledge the support of the UK Engineering and Physical Sciences Research Council (EPSRC) through the DigiTwin project (grant EP/R006768/1). 


\section{REFERENCES}

[1] N. S. Currey, Aircraft Landing Gear Design: Principles and Practices, Washington, DC, USA: American Institute of Aeronautics and Astronautics, 1988.

[2] M. Zhang, R. M. Jiang, and H. Nie, "Design and Test of Dual Actuator Nose Wheel Steering System for Large Civil Aircraft,” International Journal of Aerospace Engineering, no. Article ID 1626015, pp. 14, 2016.

[3] M. Dal Borgo, S. J. Elliott, M. Ghandchi Tehrani, and I. M. Stothers, "Robust estimation of wheel direction in ground-steering systems for aircraft," in Proceedings of the 26th International Congress on Sound and Vibration (ICSV26), Montreal, Canada, 2019, pp. 1-8.

[4] L. Jaulin, M. Kieffer, O. Didrit, and E. Walter, Applied interval analysis: with examples in parameter and state estimation, robust control and robotics, London: Springer-Verlag, 2001.

[5] G. Blewitt, "Basics of the GPS technique: observation equations," Geodetic applications of GPS, pp. 10-54: Swedish Land Survey, 1997.

[6] R. B. Langley, "The mathematics of GPS,” GPS world, vol. 2, no. 7, pp. 45-50, 1991.

[7] P. J. Huber, and E. M. Ronchetti, Robust statistics: John Wiley \& Sons, 2009.

[8] M. Oosterom, R. Babuska, and H. B. Verbruggen, "Soft computing applications in aircraft sensor management and flight control law reconfiguration,” IEEE Transactions on Systems, Man, and Cybernetics, Part C (Applications and Reviews), vol. 32, no. 2, pp. 125-139, 2002.

[9] D. Berdjag, A. Zolghadri, J. Cieslak, and P. Goupil, "Fault detection and isolation for redundant aircraft sensors," in 2010 Conference on Control and Fault-Tolerant Systems (SysTol), Nice, France, 2010, pp. 137-142. 